\title{
Adaptive Regulation of a Class of Uncertain Nonlinear Systems with Nonstrict Feedback Form
}

\author{
Jian-xiong Li, ${ }^{1,2}$ Chong-yi Gao, ${ }^{2}$ Yi-ming Fang, ${ }^{1,2}$ Cui Guo, ${ }^{1}$ and Wen-bo Zhang ${ }^{1}$ \\ ${ }^{1}$ Key Laboratory of Industrial Computer Control Engineering of Hebei Province, Yanshan University, Qinhuangdao, \\ Hebei Province 066004, China \\ ${ }^{2}$ National Engineering Research Center for Equipment and Technology of Cold Strip Rolling, Qinhuangdao, \\ Hebei Province 066004, China
}

Correspondence should be addressed to Jian-xiong Li; jxli@ysu.edu.cn

Received 30 August 2016; Accepted 31 October 2016

Academic Editor: Thierry Floquet

Copyright (C) 2016 Jian-xiong Li et al. This is an open access article distributed under the Creative Commons Attribution License, which permits unrestricted use, distribution, and reproduction in any medium, provided the original work is properly cited.

\begin{abstract}
This paper focuses on the semiglobal stabilization for a class of nonlinear systems with nonstrict feedback form. Based on a generalized scaling technique, an adaptive control algorithm with dynamic high gain is developed for a class of nonstrict feedback nonlinear systems. It can be proved that, under some appropriate design parameters, all signals of the resulting closed-loop system are bounded semiglobally, and the system state will be convergent to origin exponentially. Finally, a numerical simulation is provided to confirm the effectiveness of the proposed method.
\end{abstract}

\section{Introduction}

In this paper, we consider a class of nonlinear systems as follows:

$$
\begin{aligned}
\dot{x}_{i} & =x_{i+1}+\phi_{i}(t, x), \quad i=1, \ldots, n-1, \\
\dot{x}_{n} & =g(t, x) u+\phi_{n}(t, x),
\end{aligned}
$$

where $x=\left[x_{1}, \ldots, x_{n}\right]^{T}$ is the system state and $u$ is the control input. $g(t, x): R \times R^{n} \rightarrow R, \phi_{i}(t, x): R \times R^{n} \rightarrow R, i=$ $1, \ldots, n$, are uncertain continuous functions with $g(t, \cdot) \neq 0$ and $\phi_{i}(t, 0)=0, i=1, \ldots, n$.

System (1) can be stabilized by numerous adaptive controllers by means of backstepping technique [1-3] if (1) is of a strict feedback structure; that is, in the $i$ th subsystem, the uncertain nonlinear function $\phi_{i}(t, x)$ is the function with respect to (w.r.t.) the first state variables $x_{1}, \ldots, x_{i}$ and time $t$. Furthermore, if the function $\phi_{i}(t, x)$ depends on whole state variables but its bounding function is assumed to be a function of $x_{1}, \ldots, x_{i}$ and $t$, thus system is called semistrict feedback systems, and the backstepping technique is also an alternative method to controller design [4-7]. However, when the subsystem functions contain whole state variables and the above assumption of semistrictness is removed, that is, system (1) is of a nonstrict feedback structure, the aforementioned control methodology would be invalidated.

The control problem of nonstrict feedback nonlinear systems arises and attracts some researchers' attention. In $[8,9]$, by utilizing the monotonically increasing property of the bounding functions, the authors developed the variables separation technique and by means of the approximation property of fuzzy logic systems and neural networks (NN) proposed adaptive fuzzy and $\mathrm{NN}$ control design methods, respectively. Subsequently, the design method was extended and applied to MIMO nonlinear systems [10], stochastic nonlinear systems [11, 12], and uncertain switched nonlinear systems [13]. Furthermore, [14] eliminated the assumption in [8-13] that the unknown nonlinear functions must satisfy the monotonically increasing property of the bounding functions.

Different from [8-14], a semiglobal output feedback controller with high gain for a class of nontriangular nonlinear systems was proposed in [15], in which the authors introduced dilation $\delta_{\varepsilon}(x)=\left(x_{1}, \varepsilon x_{2}, \ldots, \varepsilon^{n-1} x_{n}\right)$ with constant 
$\varepsilon \geq 1$ and assumed that the nonlinear functions $\phi_{i}(t, x)^{\prime} \mathrm{s}$ should satisfy the following inequalities:

$$
\phi_{i}\left(t, \delta_{\varepsilon}(x)\right) \leq \varepsilon^{i-1+\sigma} c_{i}(x) \sum_{j=1}^{n}\left|x_{j}\right|, \quad i=1, \ldots, n,
$$

where $\sigma \in[0,1)$ and continuous function $c_{i}(x) \geq 0$ were known. In [15], the up-bounds of some terms containing $c_{i}(x)$ 's, such as $d_{2}$ and $d_{3}$, should be known, and these up-bounds might be very large, so that a larger gain $L$ would be required to guarantee the stability of the system. Consequently, the amplitude of the control input $u$ would be very large. Besides, the large high gain would amplify the noise [16]. On the other hand, in fact, the high gain sometimes need not be larger than the up-bounds but be just larger than the terms. To determine the gain is not easy; a trial-and-error method is often adopted [17]. Different from the constant high gain used in controller design in [15], a dynamic high gain is employed in this paper. The dynamic high gain will increase until it is larger than the terms and the closed system is stable. It can be found that the dynamic high gain or the so-called adaptive high gain was employed in [7] for dealing with the unknown growth rate of nonlinear functions and was used to avoid amplifying the noise in Extended Kalman Filters in [16].

In this paper, we focus on the stabilization scheme via state feedback for uncertain nonstrict feedback nonlinear system (1). Inspired by the generalized scaling technique of [18], a dynamic high-gain-based adaptive controller is designed for system (1), which can guarantee that all signals of the resulting closed-loop system are bounded semiglobally and the system state is convergent to the origin exponentially.

The rest of this paper is organized as follows. Two assumptions imposed on system (1) and the problem statements are presented in Section 1. The main results and theoretical analysis are given in Section 2. Simulation examples are given in Section 3, followed by Section 4 that concludes the work.

\section{Assumptions and Statements}

Rewrite system (1) as the following form:

$$
\dot{x}=A x+\Phi(t, x)+B g(t, x) u,
$$

where $\Phi(t, x)=\left[\phi_{1}(t, x), \ldots, \phi_{n}(t, x)\right]^{T} \in R^{n}$,

$$
\begin{aligned}
A & =\left[\begin{array}{ccccc}
0 & 1 & 0 & \cdots & 0 \\
0 & 0 & 1 & \cdots & 0 \\
\vdots & \vdots & \vdots & \ddots & \vdots \\
0 & 0 & 0 & \cdots & 1 \\
0 & 0 & 0 & \cdots & 0
\end{array}\right] \in R^{n \times n}, \\
B & =\left[\begin{array}{l}
0 \\
0 \\
\vdots \\
0 \\
1
\end{array}\right] \in R .
\end{aligned}
$$

And the following two assumptions are imposed on system (3).

Assumption 1. The function $g(t, x)$ is bounded and its sign is invariant and known. And there exist two known positive continuous functions $g_{m}(t, x)$ and $g_{M}(t, x)$, for all $t \geq 0$ and $x \in R$, such that

$$
0<g_{m}(t, x) \leq g(t, x) \leq g_{M}(t, x) .
$$

Without loss of generality, we suppose that $g(t, x)>0$ for all $t \geq 0$ and $x \in R^{n}$.

Assumption 2. There exist constants $\gamma_{i} \geq 0$ and $q_{i}>0$ and bounded positive continuous function $c_{i}(x), i=1, \ldots, n$, such that, for $t \geq 0, x \in R^{n}$, and $r(t) \in(1, \infty)$, the following inequality holds:

$$
\left|\phi_{i}\left(t, T_{r} x\right)\right| \leq r^{q_{i}+\gamma_{i}}(t) c_{i}(x) \sum_{j=1}^{n}\left|X_{j}\right|,
$$

where $T_{r}=\operatorname{diag}\left(r^{q_{1}}, r^{q_{2}}, \ldots, r^{q_{n}}\right) \in R^{n \times n}, q_{i}>\gamma_{i}, i=1, \ldots, n$.

Assumption 1 is given to ensure the controllability of system (3), and this assumption is generally satisfied.

Assumption 2 is about the nonlinear functions $\phi_{i}(t, x)$ 's. Compared with the corresponding assumption in [15] given by (2), the high gain $r$ in (6) is a variable rather than a constant $\varepsilon$ in (2), and, in the $i$ th inequality, the power of high gain in the right-hand side of the inequality is $q_{i}$ in (6) instead of $i-1$ in (2). It can be seen that the assumption of this paper is more general, and (2) is a special case of (6) with $q_{i}=i-1, \gamma_{i}=\sigma$, and $r(t)=\varepsilon=L$, where $L$ is an appropriate positive constant. In addition, it should be pointed out that some terms containing functions $c_{i}(x)$ 's in [15] are assumed to be bounded with up-bounds $d_{2}$ and $d_{3}$, so a large enough $L$ is required to guarantee the stability of the system. Too large gain will result in too large amplitude of the control input and will amplify the unavoidable noise [16]. Therefore, an appropriate high gain is required, and it is often achieved by using trial-and-error method [17]. In this paper, a dynamic high gain instead of constant gain will be employed in controller design.

The main objective of this paper is to design a state feedback controller $u=f_{K}(x)$ to stabilize the nonstrict feedback nonlinear system (3) under Assumptions 1 and 2.

\section{Dynamic High-Gain-Based Control and Stability Analysis}

In this section, the main result of this paper is given, an adaptive controller with dynamic high gain is presented for system (3), and the rigorous theoretical analysis is then provided.

To achieve this, a scaling transformation $z=T_{r}^{-1} x$ is first introduced, and system (3) can be changed into the following:

$$
\dot{z}=A_{r} z+\widetilde{\Phi}(t, x)+B v(t, x, u)-\frac{\dot{r}}{r} D_{q} z,
$$


where $A_{r}=A \Lambda_{r}, \widetilde{\Phi}(t, x)=T_{r}{ }^{-1} \Phi(t, x)=\left[\widetilde{\phi}_{1}(t, x), \ldots\right.$, $\left.\tilde{\phi}_{n}(t, x)\right]^{T}, v(t, x, u)=\gamma^{-q_{n}} g(t, x) u, \Lambda_{r}=\operatorname{diag}\left(r^{q_{0}}, r^{q_{2}-q_{1}}\right.$, $\left.\ldots, r^{q_{n}-q_{n-1}}\right), q_{0}>0$, and $D_{q}=\operatorname{diag}\left(q_{1}, q_{2}, \ldots, q_{n}\right)$.

Noting $x=T_{r} z$, inequality (6) in Assumption 2 imposed on system (1) can be written as follows:

$$
\begin{aligned}
\tilde{\phi}_{i}(t, x) & =r^{-q_{i}} \phi_{i}\left(t, T_{r} z\right) \leq r^{\gamma_{i}} c_{i}(z) \sum_{j=1}^{n}\left|z_{j}\right| \\
& \leq r^{\gamma_{i}} c_{i}(z) \sqrt{n}\|z\|, \quad i=1, \ldots, n .
\end{aligned}
$$

The controller $v$ is designed as

$$
v(t, x, u)=K \Lambda_{r} z
$$

where $K=\left[k_{1}, \ldots, k_{n}\right]$.

Proposition 3. Under Assumption 2, if there exist positive definite symmetric (PDS) matrices $P, Q_{1}$, and $Q_{2}$, that is, $P=$ $P^{T}>0, Q_{1}=Q_{1}{ }^{T}>0$, and $Q_{2}=Q_{2}{ }^{T} \geq 0$, such that

$$
\begin{array}{r}
(A+B K)^{T} P+P(A+B K)=-Q_{1}, \\
D_{q} P+P D_{q}=Q_{2}
\end{array}
$$

hold, and if we choose an appropriate gain $r$, then controller (9) can stabilize the state of system (7) exponentially.

Proof. Choose the positive definite function $V(x)=z^{T} P z$, and its time derivative along with (7) and (9) is derived as

$$
\begin{gathered}
\dot{V}=z^{T}\left[\Lambda_{r}(A+B K)^{T} P+P(A+B K) \Lambda_{r}\right] z \\
+2 z^{T} P \widetilde{\Phi}(t, x)-\frac{\dot{r}}{r} z^{T}\left(D_{q} P+P D_{q}\right) z .
\end{gathered}
$$

By (8), the third term in the right-hand side of (12) satisfies

$$
2 z^{T} P \widetilde{\Phi}(t, x) \leq r^{\gamma_{\max }} n\|P\|\|c(z)\|\|z\|^{2}
$$

where $\gamma_{\max }=\max \left\{\gamma_{i}\right\}, i=1,2, \ldots, n, c(z)=\left[c_{1}\left(z_{1}\right)\right.$, $\left.c_{2}\left(\bar{z}_{2}\right), \ldots, c_{n}(z)\right]^{T} \in R^{n}$.

Substituting (10), (11), and (13) into (12), it follows that

$$
\begin{aligned}
\dot{V} & \leq-z^{T} \Lambda_{r} Q_{1} z+r^{\gamma_{\max }} n\|P\|\|c(z)\|\|z\|^{2}-\frac{\dot{r}}{r} z^{T} Q_{2} z \\
& \leq-\eta\left(r^{\rho}-\frac{n \lambda_{\max }(P)\|c(z)\|}{\lambda_{\min }\left(Q_{1}\right)}\right)\|z\|^{2},
\end{aligned}
$$

where $q_{\min }=\min \left\{q_{0}, q_{2}-q_{1}, \ldots, q_{n}-q_{n-1}\right\}, \eta=\lambda_{\min }\left(Q_{1}\right) r^{\gamma_{\max }}$, $\rho=q_{\min }-\gamma_{\max }$, and $\lambda_{\min }(*)$ and $\lambda_{\text {max }}(*)$ denote the minimum eigenvalue and the maximum eigenvalue of the matrix $*$, respectively.

Let $\theta(z)=\left[n \lambda_{\max }(P)\|c(z)\| / \lambda_{\min }\left(Q_{1}\right)\right]^{1 / \rho}$ and $\theta_{\varepsilon}(z)=$ $\theta(z)+\varepsilon$, where $\varepsilon>0$. We choose $q_{i+1}>q_{i}$ and $q_{\text {min }}>\gamma_{\text {max }}$, $i=1,2, \ldots, n-1$, which means $\rho>0$. When we choose $r \geq \theta_{\varepsilon}(z)$, we have

$$
\dot{V} \leq-\eta \varepsilon_{\rho}\|z\|^{2}
$$

where $\varepsilon_{\rho} \geq \theta_{\varepsilon}^{\rho}-\theta^{\rho}>0$

Therefore, the state $z$ of system (7) will converge to origin exponentially. It should be pointed out that, in Assumption 2, $c_{i}(x)$ 's are supposed to be bounded, but we do not know the up-bounds of $c_{i}(x)$ 's, so the closed-loop system (7) and (9) is semiglobally stable. The proof is thus completed.

The real controller $u$ can be directly obtained from (9) as the following form:

$$
u=\frac{r^{q_{n}}}{g(t, x)} K \Lambda_{r} z .
$$

However, controller (16) is invalid because $g(t, x)$ is unknown. Under Assumption 1, the bounds of $g(t, x)$ could be used instead of $g(t, x)$; the controller is designed as

$$
u=\frac{r^{q_{n}}}{g_{m}(t, x)} K \Lambda_{r} z
$$

And it should be pointed out that, in Proposition 3, the high gain $r$ should be equal to or larger than $\bar{\theta}_{\varepsilon}(z)$ to guarantee the convergence of $z$. If a constant gain $r$ is chosen, for example, in [15], the bound of $\|c(z)\|$ should be known, and a very large gain is required; meanwhile, with higher $r$, more noise is amplified [16]. And, from (17), it is easy to see that large $r$ will bring about large control input $u$ and may lead to the control input saturation due to physical limit in practice. To reduce this defect of large high gain, a dynamic high gain is employed in this paper, and the update law of the high gain $r$ is given as follows:

$$
\dot{r}=\frac{\alpha}{r}\left(\bar{\theta}_{\varepsilon}(z)-r\right) z_{1}^{2}, \quad r(0) \geq 1 .
$$

From (18), it is obvious that $\gamma$ is increasing until $r \geq \bar{\theta}_{\varepsilon}(z)$ or $z_{1} \equiv 0$.

Theorem 4. Under Assumptions 1 and 2, if there exist PSD matrices $P, Q_{1}$, and $Q_{2}$, such that (10) and (11) hold, controller (17) with gain update law (18) can guarantee that all the signals of the closed-loop system are uniformly ultimately bounded and the states are convergent to origin exponentially.

Proof. Choose the positive definite function $V(x)=z^{T} P z$, and its time derivative along with (7) and (17) is derived as

$$
\begin{aligned}
\dot{V}= & z^{T}\left[\Lambda_{r g}(A+B K)^{T} P+P(A+B K) \Lambda_{r g}\right] z \\
& +2 z^{T} P T_{r}^{-1} \Phi(t, x)-\frac{\dot{r}}{r} z^{T}\left(D_{q} P+P D_{q}\right) z,
\end{aligned}
$$

where $\Lambda_{r g}=\operatorname{diag}\left(1, \ldots, 1, g_{m}{ }^{-1} g\right) \Lambda_{r}$.

Substituting (10), (11), and (13) into (19) and noting $g_{m} g(t, x) \geq 1$, we get

$$
\begin{aligned}
\dot{V} & \leq-z^{T} \Lambda_{r g} Q_{1} z+r^{r_{\max }} n\|P\|\|c(z)\|\|z\|^{2}-\frac{\dot{r}}{r} z^{T} Q_{2} z \\
& \leq-\eta\left(r^{\rho}-\frac{n \lambda_{\max }(P)\|c(z)\|}{\lambda_{\min }\left(Q_{1}\right)}\right)\|z\|^{2} .
\end{aligned}
$$


Following the proof of Proposition 3, when $r \geq \bar{\theta}_{\varepsilon}(z)$, we have $\dot{V} \leq-\eta \varepsilon_{\rho}\|z\|^{2}$, which implies that the state $z$ of system (7) will converge to origin exponentially.

The next step is to illustrate the boundedness of the high gain $r$.

Firstly, we suppose $z_{1} \neq 0$ at time $t \in\left[t_{0}, t_{f}\right]$, where $t_{0}$ and $t_{f}$ are initial time and final time, respectively, and $t_{f} \epsilon$ $\left(t_{0}, \infty\right)$. In this case, we can get $\dot{r} \geq 0$ when $r \leq \bar{\theta}_{\varepsilon}(z)$ and $\dot{r}<0$ when $r>\bar{\theta}_{\varepsilon}(z)$, which implies that $r$ is bounded; that is, there exists an arbitrarily small positive constant $\varepsilon^{\prime}$; the inequality $r \leq \bar{\theta}_{\varepsilon}(z)+\varepsilon^{\prime}$ holds.

In the case of $z_{1} \equiv 0$, it follows that $\dot{r}=0$ regardless of whether $r \leq \bar{\theta}_{\varepsilon}(z)$ or not, which also means $r$ is bounded.

Then, we can obtain that the high gain $r$ is bounded.

To sum up, we can conclude that all the signals of the closed-loop system of (6), (17), and (18) are bounded, and the system state $x$ converges to origin exponentially. That completes the proof.

\section{Numerical Simulation}

To illustrate the effectiveness of the proposed method, a numerical simulation is presented in this section.

We consider the following nonlinear system:

$$
\begin{aligned}
& \dot{x}_{1}=x_{2}+x_{1}^{2 / 3} x_{2}^{1 / 3}, \\
& \dot{x}_{2}=x_{3}+x_{1} x_{3}^{1 / 3} \ln \left(1+x_{2}^{2}\right), \\
& \dot{x}_{3}=\left(2+\sin x_{2}\right)\left(1+x_{1}^{2}\right) u+x_{1} .
\end{aligned}
$$

It can be verified that system (21) satisfies Assumption 1 with $g_{m}=\left(1+x_{1}{ }^{2}\right)$ and $g_{M}=3\left(1+x_{1}{ }^{2}\right)$ and satisfies Assumption 2 with $q_{0}=1, q_{1}=0, q_{2}=1, q_{3}=3, \gamma_{1}=1 / 3$, $\gamma_{2}=0.5, \gamma_{3}=0, c_{1}(z)=c_{3}(z)=1$, and $c_{2}(z)=\left|z_{1}\right|^{1 / 3}\left(2+z_{2}{ }^{2}\right)$.

The controller parameter of (17) and parameters of (18) are chosen as $K=[-4,-5,-3], \alpha=0.5$, and $\varepsilon=0.01$. Choosing $Q_{1}=\operatorname{diag}(0.5,0.5,0.5)$ and solving $(10)$, we get

$$
P=\left[\begin{array}{ccc}
0.2989 & -0.1569 & 0.0414 \\
-0.1569 & 0.3071 & -0.2874 \\
0.0414 & -0.2874 & 1.8258
\end{array}\right]
$$

And, from (11), we have

$$
Q_{2}=\left[\begin{array}{ccc}
1.1955 & -1.0982 & 0.3724 \\
-1.0982 & 3.0709 & -3.4491 \\
0.3724 & -3.4491 & 25.5613
\end{array}\right] \text {. }
$$

Furthermore, we can get $\lambda_{\text {max }}(P)=1.8814, \lambda_{\text {min }}\left(Q_{1}\right)=0.5$, $q_{\text {min }}=1, \gamma_{\max }=0.5$, and $\rho=0.5$.

The simulations are run under the initial conditions $x_{0}=$ $[2,0,0]^{T}$ and three different values of $r(0)$; that is, $r(0)=1,3$, and 6 .
The simulation results are shown in Figures 1-3. It is clearly shown that, under the action of the proposed control (17) and adaptive law (18), all the signals are bounded and the system states are convergent to origin.

Furthermore, it is easy to see that when larger $r(0)$ is chosen, $x(t)$ is driven faster to steady state (see Figure 1); correspondingly, $u(t)$ and $r(t)$ achieve steady state more quickly (see Figures 2 and 3 ).

And, from the definition of $\theta_{\varepsilon}(z)$ and $\theta(z)$, it can be calculated that $\theta_{\varepsilon}(z(0))=5.7213$; according to (18), if $r(0)<$ 5.7213, $\left.\dot{r}\right|_{t=0}>0$; otherwise, $\left.\dot{r}\right|_{t=0} \leq 0$; therefore, in Figure 3, we can see that, at the beginning, $r(t)$ increases when $r(0)=1$ and $r(0)=3$, while $r(t)$ decreases when $r(0)=6$.

In practice, the control input is often limited due to some physical limits. Too large high gain could lead to the control input saturation, which would degrade the performance of the control system and even cause system instability. To illustrate the above problem caused by too large high gain, some more simulations are given as follows.

From Figure 3, the high gain $r$ can be approximately regarded as a constant, $r=6$. And if a constant high gain is employed in the controller design, to ensure the stability of the system, $r=6$ or a larger one would be chosen using trialand-error strategy. So, the simulations are run with the same design parameters and the same initial condition of state and $r(0)=6$. And two cases of the control input saturation are considered; namely, $|u| \leq 65$ and $|u| \leq 63$. The simulation results are shown in Figures 4 and 5.

In comparison between the state responses with $r(0)=6$ in Figure 1 and the state responses in Figure 4, it can be found that the performance of the control system degrades when the control input is restricted by $|u| \leq 65$ but is still stable, while the system would be unstable when the control input is limited by $|u| \leq 63$.

Under the control input limit $|u| \leq 63$, the controller with constant high gain $r=6$ or a larger one will be invalid. But a small gain cannot ensure the stability of the system. To solve the above problem, this paper gives an alternative method. We can find that if we choose $r(0)=1$, the input saturation does not occur (see Figure 2(a)). Therefore, a smaller $r(0)$ will bring a smaller control input and will avoid the input saturation.

\section{Conclusions}

In this paper, an adaptive controller with dynamic high gain has been designed for a class of nonstrict feedback nonlinear systems. The main difference from the existing related works is that a dynamic high gain rather than a constant one is employed in the controller design. The advantage of the dynamic high gain is that it would not result in a large control input, and it is more likely to avoid the input saturation occurring which usually exists due to physical limits in practice. By Lyapunov stability theory method, a rigorous theoretical proof is provided to show that the proposed controller can guarantee the semiglobal stability of the system state. Finally, a numerical simulation is provided to illustrate the effectiveness of the proposed method. 


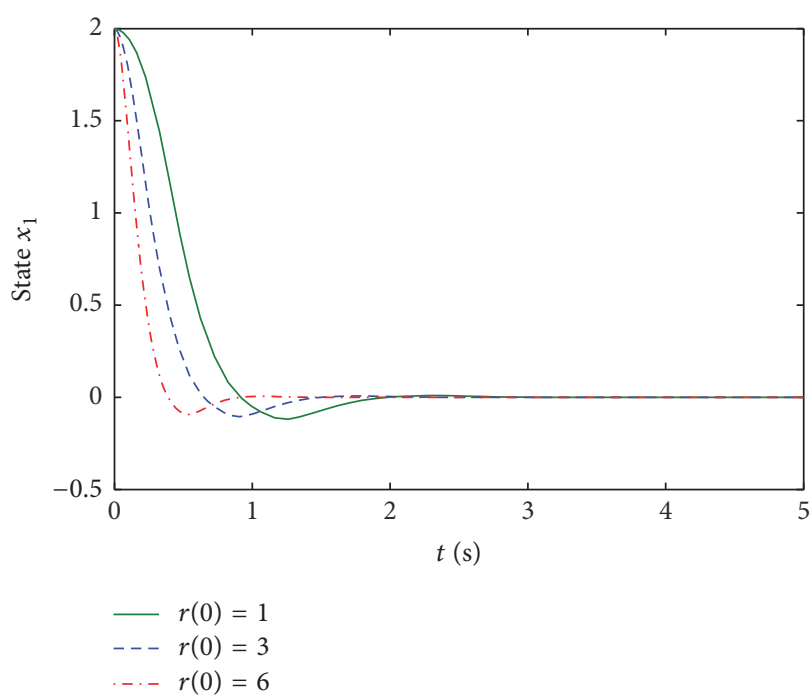

(a)

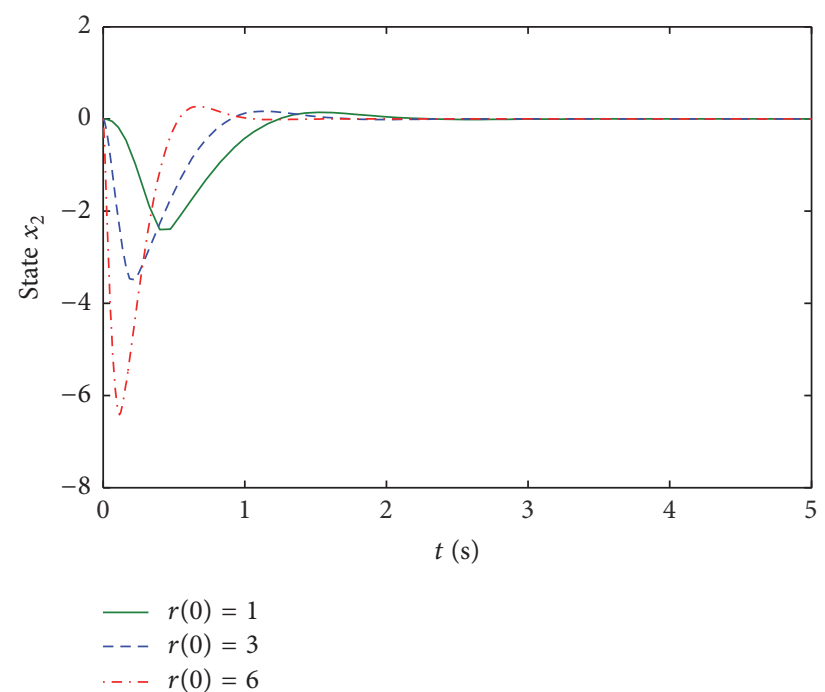

(b)

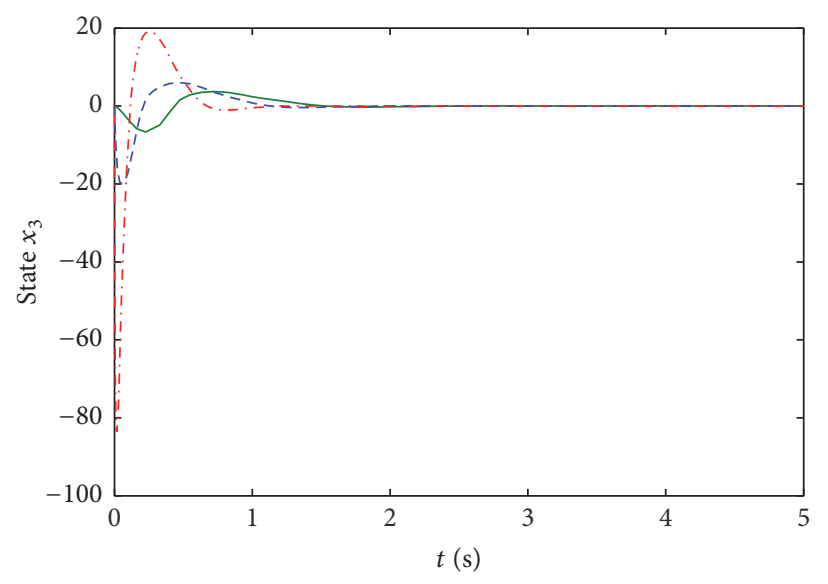

$\begin{aligned}-r(0) & =3 \\ ---r(0) & =6\end{aligned}$

-.- $r(0)=6$

(c)

FIGURE 1: State response.

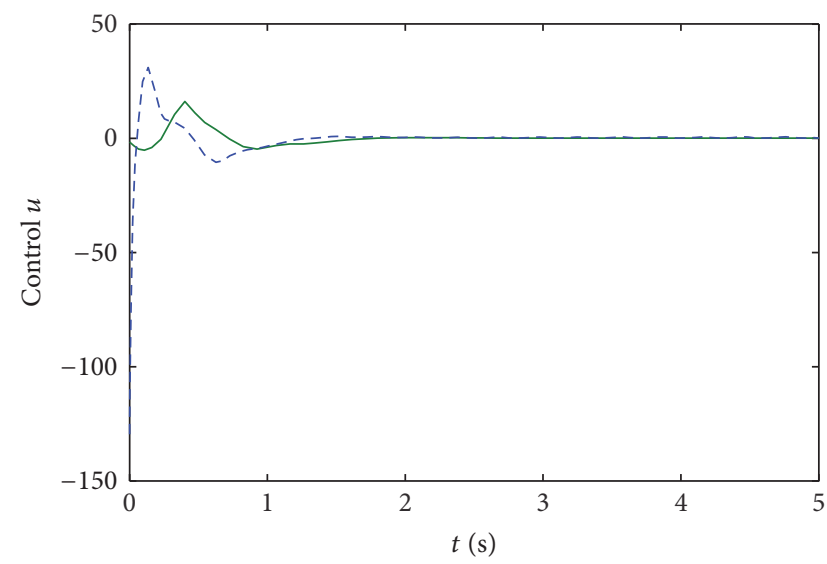

$r(0)=1$

$--r(0)=3$

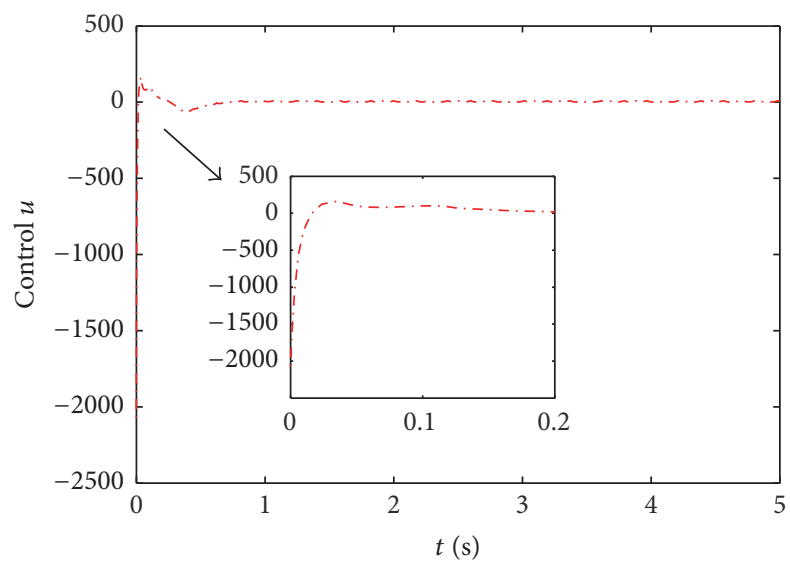

$r(0)=6$

FIgURE 2: Control input $u$. 


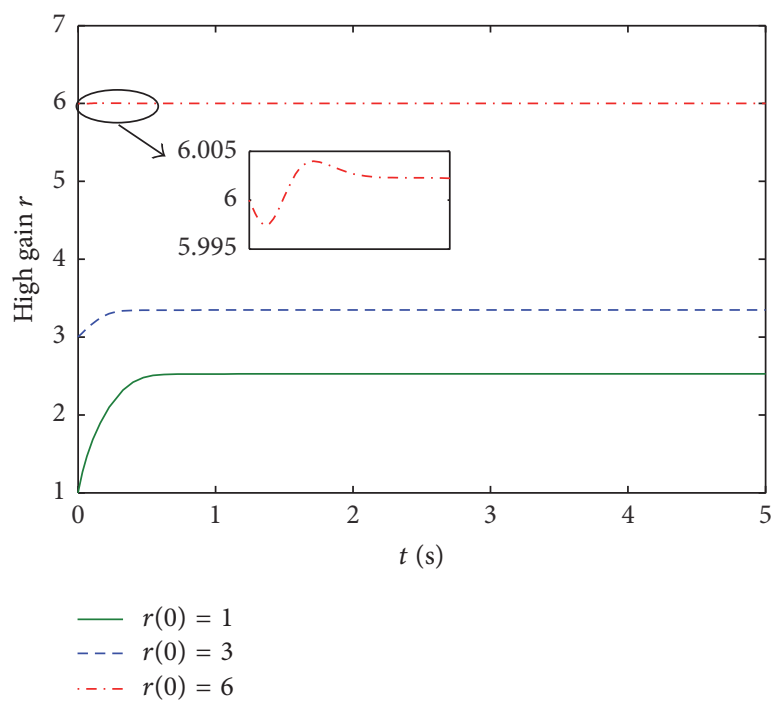

Figure 3: High gain $r$.

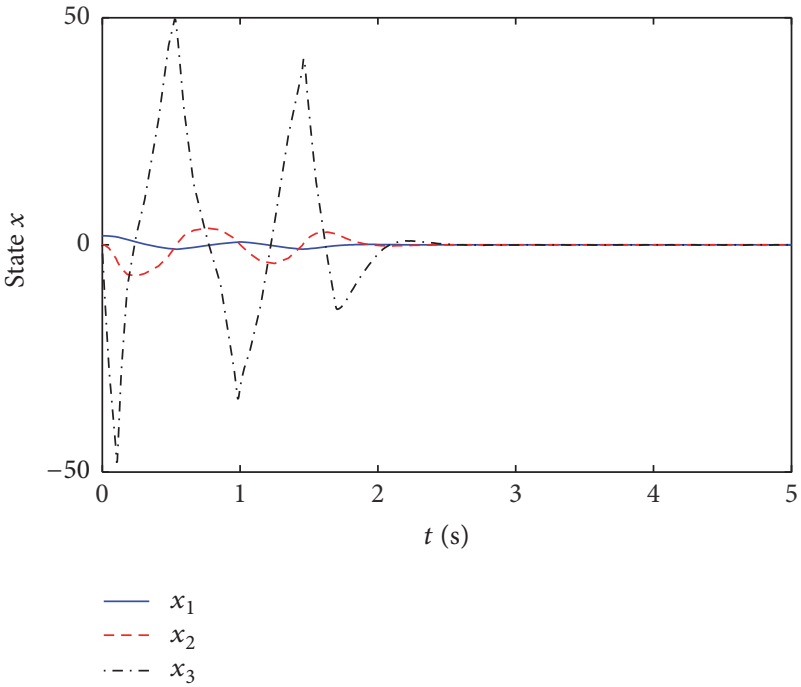

(a) $|u| \leq 65$

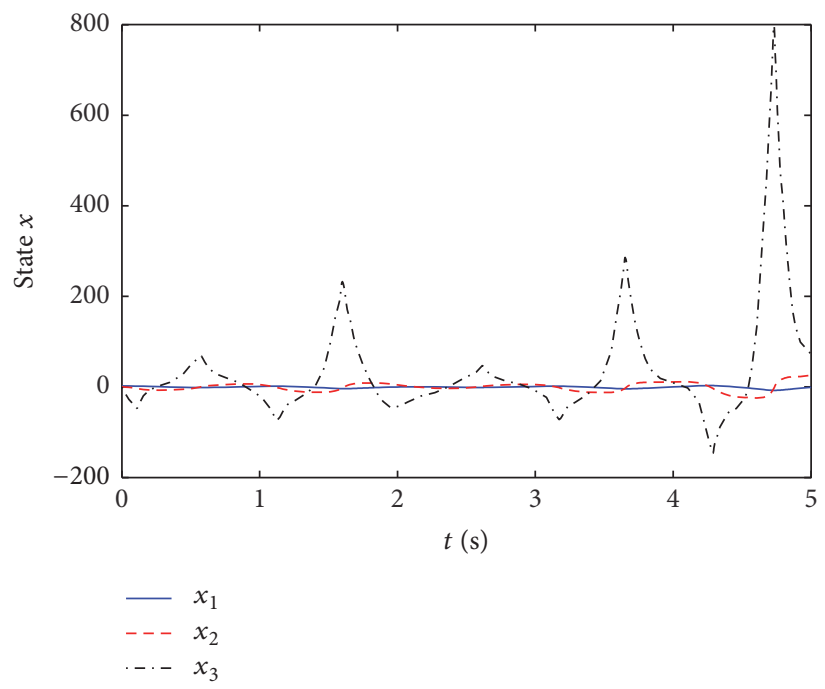

(b) $|u| \leq 63$

Figure 4: State response with input saturation and $r(0)=6$.

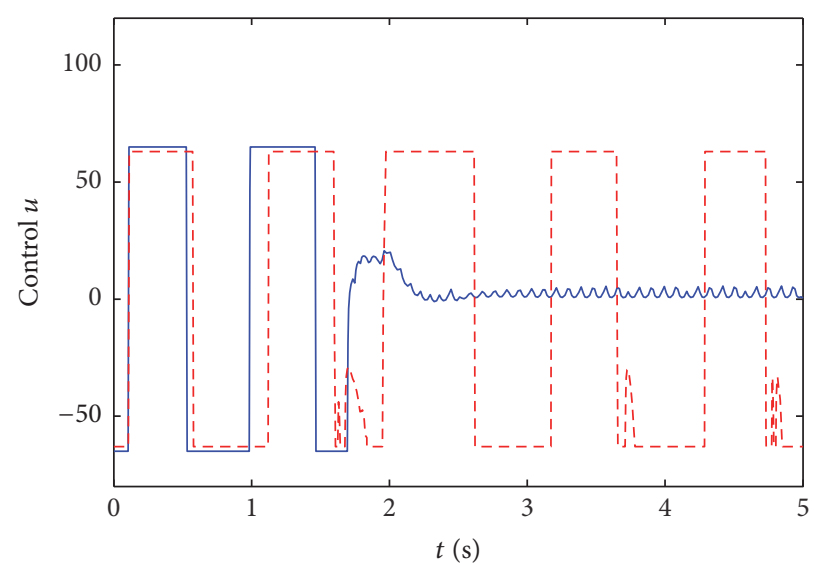

With input saturation $|u| \leq 65$

With input saturation $|u| \leq 63$

FIGURE 5: Control input with input saturation and $r(0)=6$. 


\section{Competing Interests}

The authors declare that there are no competing interests regarding the publication of this paper.

\section{Acknowledgments}

This research was supported in part by the National Natural Science Foundation of China under Grant 61403332, in part by the Natural Science Foundation of Hebei Province under Grant F2015203400, in part by the National Natural Science Foundation of China and Baosteel Group Co. Ltd. under Grant U1260203, and in part by the Higher Education Innovation Team of Hebei Province Leading Talent Cultivation Project under Grant LJRC013.

\section{References}

[1] A. Saberi, P. V. Kokotovic, and H. J. Sussmann, "Global stabilization of partially linear composite systems," SIAM Journal on Control \& Optimization, vol. 28, no. 6, pp. 1491-1503, 1990.

[2] P. P. Yip and J. K. Hedrick, "Adaptive dynamic surface control: a simplified algorithm for adaptive backstepping control of nonlinear systems," International Journal of Control, vol. 71, no. 5, pp. 959-979, 1998.

[3] K. K. Ahn, D. N. C. Nam, and M. Jin, "Adaptive backstepping control of an electrohydraulic actuator," IEEE/ASME Transactions on Mechatronics, vol. 19, no. 3, pp. 987-995, 2014.

[4] B. Yao and M. Tomizuka, "Adaptive robust control of SISO nonlinear systems in a semi-strict feedback form," Automatica, vol. 33, no. 5, pp. 893-900, 1997.

[5] J. Q. Gong and B. Yao, "Neural network adaptive robust control of nonlinear systems in semi-strict feedback form," Automatica, vol. 37, no. 8, pp. 1149-1160, 2001.

[6] Y.-S. Lin and C.-C. Cheng, "Design of terminal block backstepping controllers for perturbed systems in semi-strict feedback form," International Journal of Control, vol. 88, no. 10, pp. 21072116, 2015.

[7] F. Li and Y. Liu, "Global stabilization via time-varying outputfeedback for stochastic nonlinear systems with unknown growth rate," Systems and Control Letters, vol. 77, pp. 69-79, 2015.

[8] B. Chen, X. P. Liu, S. S. Ge, and C. Lin, "Adaptive fuzzy control of a class of nonlinear systems by fuzzy approximation approach," IEEE Transactions on Fuzzy Systems, vol. 20, no. 6, pp. 1012-1021, 2012.

[9] B. Chen, K. Liu, X. Liu, P. Shi, C. Lin, and H. Zhang, "Approximation-based adaptive neural control design for a class of nonlinear systems," IEEE Transactions on Cybernetics, vol. 44, no. 5, pp. 610-619, 2014.

[10] B. Chen, C. Lin, X. Liu, and K. Liu, "Adaptive fuzzy tracking control for a class of MIMO nonlinear systems in nonstrictfeedback form," IEEE Transactions on Cybernetics, vol. 45, no. 12, pp. 2744-2755, 2015.

[11] H. Wang, K. Liu, X. Liu, B. Chen, and C. Lin, "Neural-based adaptive output-feedback control for a class of nonstrict-feedback stochastic nonlinear systems," IEEE Transactions on Cybernetics, vol. 45, no. 9, pp. 1977-1987, 2015.

[12] H. Wang, B. Chen, K. Liu, X. Liu, and C. Lin, "Adaptive neural tracking control for a class of nonstrict-feedback stochastic nonlinear systems with unknown backlash-like hysteresis," IEEE Transactions on Neural Networks \& Learning Systems, vol. 25, no. 5, pp. 947-958, 2014.

[13] S. J. Yoo, "Adaptive tracking control for uncertain switched nonlinear systems in nonstrict-feedback form," Journal of the Franklin Institute, vol. 353, no. 6, pp. 1409-1422, 2016.

[14] S. Tong, Y. Li, and S. Sui, "Adaptive fuzzy tracking control design for uncertain non-strict feedback nonlinear systems," IEEE Transactions on Fuzzy Systems, 2016.

[15] M. Liu, Y. Liu, and F. Li, "Semiglobal stabilization via outputfeedback for a class of nontriangular nonlinear systems with an unknown coefficient," Mathematical Problems in Engineering, vol. 2015, Article ID 375765, 8 pages, 2015.

[16] N. Boizot, E. Busvelle, and J.-P. Gauthier, "An adaptive high-gain observer for nonlinear systems," Automatica, vol. 46, no. 9, pp. 1483-1488, 2010.

[17] M. Farza, M. M'Saad, T. Maatoug, and M. Kamoun, "Adaptive observers for nonlinearly parameterized class of nonlinear systems," Automatica, vol. 45, no. 10, pp. 2292-2299, 2009.

[18] P. Krishnamurthy and F. Khorrami, "Generalized state scaling and applications to feedback, feedforward, and nontriangular nonlinear systems," IEEE Transactions on Automatic Control, vol. 52, no. 1, pp. 102-108, 2007. 


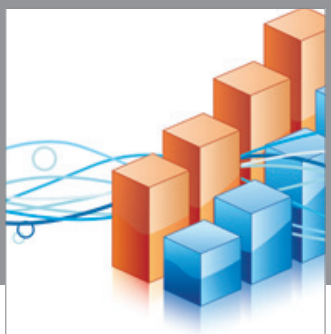

Advances in

Operations Research

vatem alat4

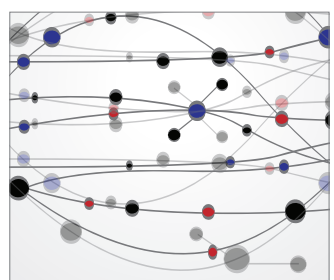

\section{The Scientific} World Journal
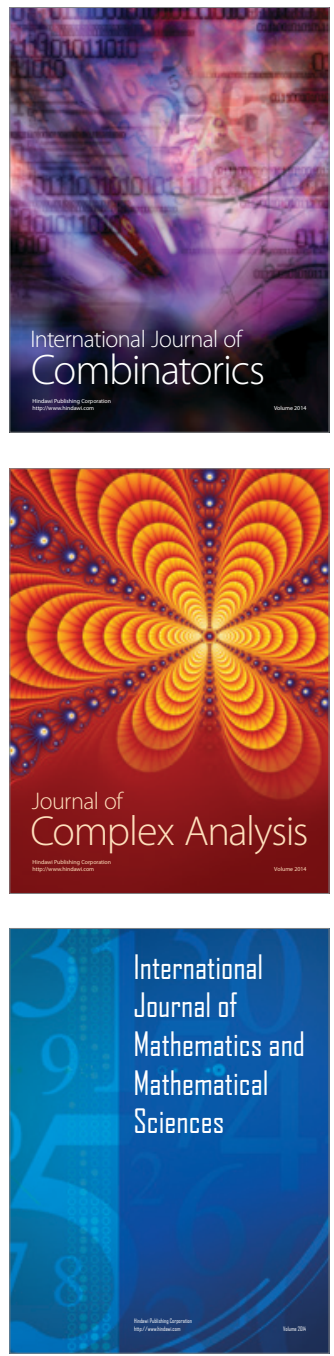
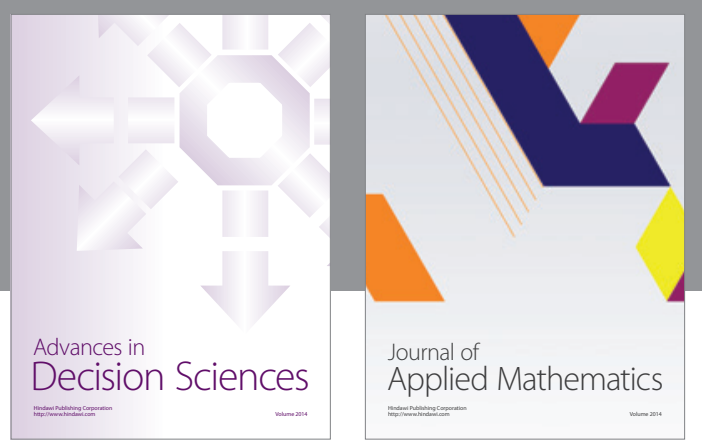

Algebra

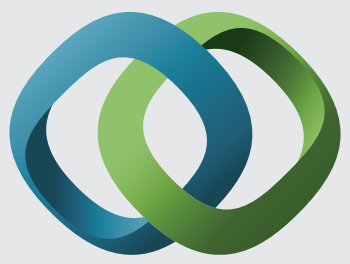

\section{Hindawi}

Submit your manuscripts at

http://www.hindawi.com
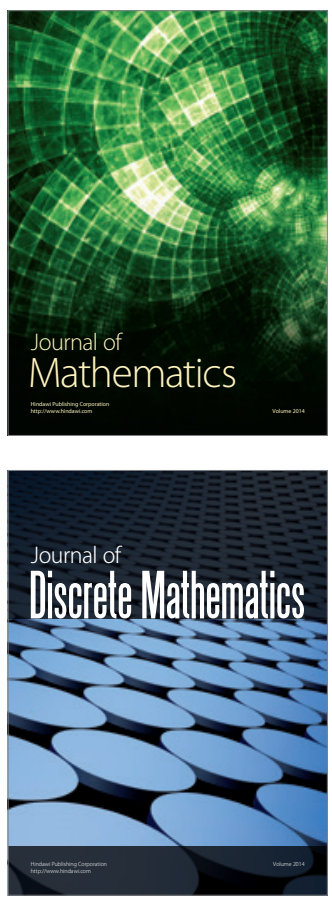

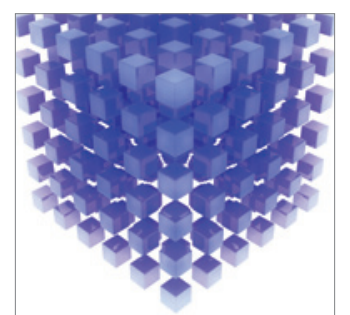

Mathematical Problems in Engineering
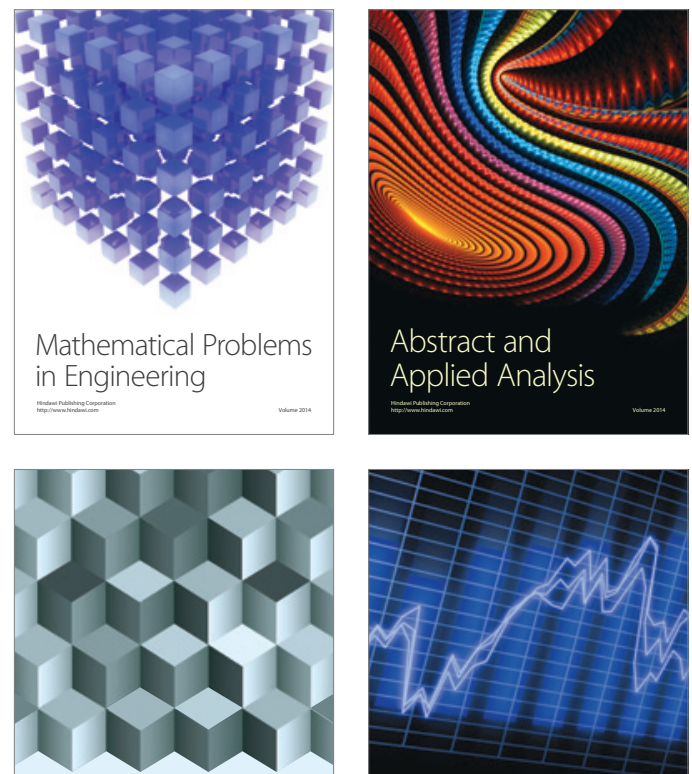

Journal of

Function Spaces

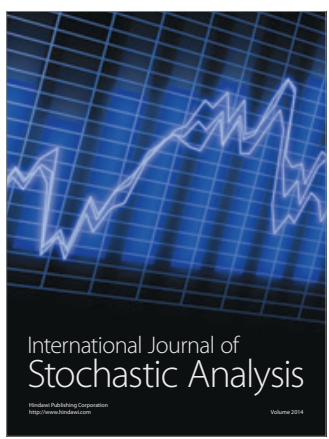

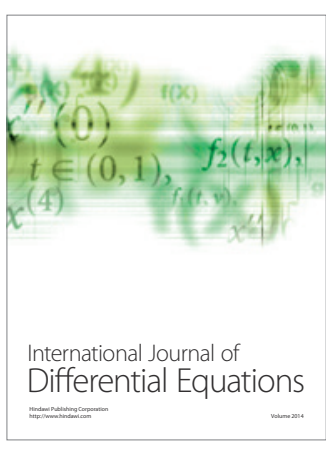
